\title{
Direct detection of Helicobacter pylori resistance to macrolides by a polymerase chain reaction/DNA enzyme immunoassay in gastric biopsy specimens
}

\author{
A Marais, L Monteiro, A Occhialini, M Pina, H Lamouliatte, F Mégraud
}

\begin{abstract}
Background-The increasing use of macrolides especially in the treatment of Helicobacter pylori infection has led to an increase in resistant strains. The resistance of $H$ pylori to macrolides, especially clarithromycin, is one of the major causes of eradication failure. In $\mathrm{H}$ pylori, clarithromycin resistance is due to point muta-
\end{abstract} tions localised in domain $\mathrm{V}$ of $23 \mathrm{~S}$ rRA.

Aim-To develop a molecular technique based on amplification of a relevant fragment of the 23S rRNA and colorimetric hybridisation in liquid phase to detect directly in biopsy specimens the type of mutation associated with resistance of $H$ pylori to clarithromycin.

Methods-Gastric biopsy samples from 61 patients were submitted to this test. The results were compared with standard methods (determination of minimal inhibition concentration, polymerase chain reaction/restriction fragment length polymorphism, and/or DNA sequencing) in order to evaluate the test and to define the cut off values, specificity, and sensitivity.

Results-The 14 biopsy samples in which $H$ pylori was not detected did not give a positive result in any assay, and the 14 samples harbouring strains susceptible to clarithromycin gave a positive result with the wild type probe as expected. The 33 biopsy specimens containing resistant strains always gave a positive signal with one of the probes detecting resistant organisms, but in eight cases they also reacted with the wild type probe, indicating that a mixture of resistant and susceptible organisms was present.

Conclusion-The importance of this new assay is that it allows the detection of multiple genotypes corresponding to either heterogeneous genotypes or mixed infections. Moreover, it allows in a single step not only the detection of $H$ pylori but also the determination of its susceptibility to clarithromycin directly in biopsy specimens without the need for culture. (Gut 1999;44:463-467)

Keywords: Helicobacter pylori; resistance; clarithromycin; macrolide; polymerase chain reaction (PCR); immunoassay

The recognition of the role of Helicobacter pylori in gastroduodenal diseases, especially peptic ulcer diseases, has led to the wide use of anti- biotics to eradicate this bacterium. Triple therapies have been advocated and result in a high cure rate. ${ }^{12}$ However, failure does occur, and resistance to antibiotics, in particular macrolides, is the leading cause of failure, and its prevalence seems to be increasing. ${ }^{3}$

The mechanism of macrolide resistance in $H$ pylori has been elucidated..$^{4-6}$ It is based on point mutations localised in the 23S rRNA peptidyltransferase domain as described in several other bacteria. ${ }^{7}$ In $H$ pylori, a point mutation in the 23S rRNA gene is so far the only mechanism of clarithromycin resistance elucidated; it prevents the binding of macrolides to the $50 \mathrm{~S}$ ribosomal subunit. ${ }^{6}$ No erm gene, a gene named for erythromycin ribosome methylation, was detected by polymerase chain reaction (PCR $)^{58}$ using conserved primers. ${ }^{9}$

Until now, $H$ pylori resistance to macrolides has been determined by phenotypic methods which take five days or more. Furthermore, they do not give any insight into the type of mutation present, which may be interesting from an epidemiological point of view. Recently, our group ${ }^{10}$ has developed a molecular method which allows the identification of the genotype associated with clarithromycin resistance of $H$ pylori. This method is based on PCR amplification of a $425 \mathrm{bp}$ fragment of the $23 \mathrm{~S}$ rRNA peptidyltransferase region ( $\mathrm{Hp} 23 \mathrm{~S}$ fragment), associated with hybridisation of the PCR product in liquid phase with internal probes specific for each resistant genotype. Despite the efficacy of this method, it still requires culture of $H$ pylori. The aim of this study was therefore to apply this technique to DNA directly amplified from biopsy specimens in order to obtain a result within a day of endoscopy.

\section{Materials and methods}

CLINICAL SAMPLES

Gastric biopsy samples from 61 patients who had not received $H$ pylori eradication treatment were obtained at the time of endoscopy. Forty seven were classified as $H$ pylori positive on the basis of culture, and 14 were found to be $H$ pylori negative and were used as negative controls in further experiments. The patients were successively included until the number with resistant strains reached 33. However, for the cases that turned out to be $H$ pylori negative as well as for those where an $H$ pylori strain

Abbreviations used in this paper: $\mathrm{MIC}$, minimal inhibition concentration; PCR, polymerase chain reaction; DEIA, DNA enzyme immunoassay; RFLP, restriction fragment length polymorphism. 
susceptible to clarithromycin was isolated, only the first 14 in each category were included. The $H$ pylori positive gastric biopsy specimens were from 20 patients with duodenal ulcer and 24 with non-ulcer dyspepsia. No clinical data could be obtained for the three remaining patients.

BACTERIAL STRAINS AND CULTURE

$H$ pylori was cultured from gastric biopsy samples ground for 2-3 seconds with an electric tissue homogeniser (Ultraturax; LaboModerne, Paris, France) before inoculation on to a selective in house medium, pylori agar (bioMérieux, Marcy l'Etoile, France), and a non-selective Columbia medium (Oxoid; Unipath, Basingstoke, Hants, UK) enriched with $10 \%$ human blood. The plates were incubated under microaerobic conditions at $37^{\circ} \mathrm{C}$ for 12 days. A subculture of $H$ pylori strains was performed under the same conditions for two to three days.

DETERMINATION OF MINIMAL INHIBITION

CONCENTRATION (MIC)

The MICs of clarithromycin on $H$ pylori strains were determined by Etest (AB Biodisks, Solna, Sweden) and by an agar dilution method, as previously described. ${ }^{6}$ The plates were incubated in a microaerobic atmosphere (jars with Gaspack $\mathrm{H}_{2}+\mathrm{CO}_{2}$ without catalyst (Oxoid)) at $37^{\circ} \mathrm{C}$ for two days. A strain was considered resistant when the MIC was higher than $2 \mathrm{mg} / \mathrm{l}$.

DNA EXTRACTION

The biopsy samples were ground as described above and centrifuged for five minutes at $10000 \mathrm{~g}$. The pellet was resuspended in $300 \mu \mathrm{l}$ extraction buffer (20 mM Tris/ $\mathrm{HCl}, \mathrm{pH} 8$, $0.5 \%$ Tween 20 ), and proteinase $\mathrm{K}$ was added at a final concentration of $0.5 \mathrm{mg} / \mathrm{ml}$. The mixture was incubated at $56^{\circ} \mathrm{C}$ for one hour. Finally, the enzyme was inactivated by boiling for 10 minutes.

AMPLIFICATION OF THE HP23S FRAGMENT

The Hp23S fragment was obtained by PCR amplification of $H$ pylori extracted DNA with primers Hp23-1 (5'-CCACAGCGATGTGG TCTCAG-3') and $\mathrm{Hp} 23-2$ (5'-CTCCAT AAGAGCCAAAGCCC-3'), which are complementary from nucleotide 1820 to 1839 and from nucleotide 2244 to 2225 respectively, as previously described, ${ }^{6}{ }^{10}$ except for the amount of Taq polymerase (2 units) (Eurobio, Les Ulis, France) and the concentration of primers (1 $\mu \mathrm{M})$ (Eurogentec, Seraing, Belgium). This fragment corresponds to the peptidyltransferase region of the $23 \mathrm{~S}$ rRNA gene. The amplified $425 \mathrm{bp}$ fragment was visualised after electrophoresis on a $1.5 \%$ agarose gel stained with ethidium bromide.

RESTRICTION FRAGMENT LENGTH POLYMORPHISM (RFLP) OF THE HP23S FRAGMENT

As previously described, ${ }^{6}{ }^{10}$ digestion of the $\mathrm{Hp} 23 \mathrm{~S}$ fragment with the restriction enzymes $B b s \mathrm{I}$ and $B s a \mathrm{I}$ permitted the discrimination between two kinds of mutant genotype (A2143G and A2144G respectively). Diges- tions were performed according to the instructions of the manufacturer (New England Biolabs Inc, Beverly, Massachusetts, USA). Restriction products were analysed on a $2 \%$ agarose gel.

SEQUENCING OF AMPLIFIED PRODUCTS

Before sequencing, the PCR products were purified by using the Wizard PCR preps (Promega, Madison, Wisconsin, USA). The sequencing reaction was performed as described by Sanger et $a l^{11}$ using a Dye Terminator Ready Reaction kit as indicated by the manufacturer (Perkin Elmer, Applied Biosystem Division, Foster City, California, USA). The same primers as were used for PCR were used for sequencing. The sequencing reaction took place in a Perkin Elmer 9600 thermal cycler. Twenty five cycles were applied, each of 10 seconds at $96^{\circ} \mathrm{C}$, five seconds at $50^{\circ} \mathrm{C}$, and four minutes at $60^{\circ} \mathrm{C}$. Sequencing was performed on the two strands of each PCR product with the automated DNA sequencer ABI Prism 377 (Perkin Elmer).

DNA ENZYME IMMUNOASSAY (DEIA)

This colorimetric hybridisation assay was performed with the GEN-ETI-K DEIA kit as previously described. ${ }^{10}{ }^{12}$ Four different 5' biotinylated probes were designed to be specific for each genotype (wild type and the three mutants A2143C, A2143G, A2144G). Optimal hybridisation conditions were previously determined. ${ }^{10}$ Data presented in this study are the means of three independent experiments.

\section{DETERMINATION OF CUT OFF VALUE}

Cut off values for the probes wild type, 2143G, and $2144 \mathrm{G}$ were determined by the receiving operating characteristics curve construction method $^{13}$ as previously described. ${ }^{10}{ }^{12}$ Briefly, different virtual cut off values between 0 and 4 (OD) were assigned, and for each cut off value, the numbers of true positives, true negatives, false positives, and false negatives were calculated in comparison with the standard method. Thus each cut off gives a sensitivity and a specificity value. These data are then used to determine the cut off value that provides both the best sensitivity and the best specificity.

Owing to the low number of positive cases for the $2143 \mathrm{C}$ probe, the cut off value was arbitrarily defined as the mean of the values corresponding to a negative result by the reference method plus three standard deviation values.

\section{Results}

AMPLIFICATION OF THE HP23S FRAGMENT

Amplification with DNA extracted from biopsy samples for which $H$ pylori culture was positive yielded a fragment of the expected size. In contrast, no fragment was obtained with DNA from $H$ pylori negative biopsy samples (data not shown). The specificity of the amplification with primers Hp23-1 and Hp23-2 has previously been shown. ${ }^{6}{ }^{10}$ The patients were not selected on the basis of the CLOtest nor heavy growth. There was complete correlation 
Table 1 Cut off values, sensitivity, and specificity of the four probes used to detect resistance of Helicobacter pylori to macrolides with the DNA enzyme immunoassay

\begin{tabular}{llll}
\hline Probe & Cut off value & Sensitivity (\%) & Specificity (\%) \\
\hline WT & 0.9 & 100 & 97.6 \\
$2143 \mathrm{C}$ & 0.96 & ND & ND \\
$2143 \mathrm{G}$ & 0.6 & 100 & 100 \\
$2144 \mathrm{G}$ & 0.6 & 100 & 97.5
\end{tabular}

The cut off values were defined by using receiving operating characteristics curves, except for the $2143 \mathrm{C}$ probe (mean $+3 \mathrm{SD})$

WT, wild type; ND, not determined.

between culture and PCR results. We compared the semiquantitative results of culture $(+,++,+++)$ with the OD obtained by DEIA for each probe when a positive result was obtained. No correlation was found (data not shown).

The reproducibility of the three OD measurements was satisfactory, with standard deviations of less than $10 \%$ of the mean and even less than $4 \%$ for probes $2144 \mathrm{G}$ and $2143 \mathrm{C}$.
DETERMINATION OF THE CUT OFF VALUES

The receiving operating characteristic curves were constructed for the wild type, $2143 \mathrm{G}$, and $2144 \mathrm{G}$ probes. They were close to the ideal curves. The cut off value for the $2143 \mathrm{C}$ probe was 0.967 (mean of negative cases 0.746 plus three standard deviations). Table 1 gives the cut off values as well as the sensitivity and specificity for each probe.

DISTRIBUTION OF DIFFERENT 23S rDNA GENOTYPES DIRECTLY DETECTED IN BIOPSY SPECIMENS

The results from the DEIA were analysed on the basis of the cut off values defined above. They were compared with the results obtained from MIC determination, PCR/RFLP, and/or PCR product sequencing performed on the strains isolated from the corresponding biopsies (table 2). The Etest was used as a screening test, and the agar dilution test was

Table 2 Comparison of the results obtained by the three reference methods and the DNA enzyme immunoassay (DEIA) to detect Helicobacter pylori resistance to macrolide

\begin{tabular}{|c|c|c|c|c|c|c|c|}
\hline \multirow[b]{2}{*}{$\begin{array}{l}\text { Biopsy } \\
\text { sample }\end{array}$} & \multicolumn{7}{|c|}{ Resistance determined by } \\
\hline & $M I C(m g / l)$ & $P C R / R F L P$ & Sequence analysis * & $\begin{array}{l}D E I A \\
W T\end{array}$ & $2143 C$ & $2143 G$ & $2144 G$ \\
\hline 1 & $>128$ & NR & $2143 \mathrm{C}$ & - & + & - & - \\
\hline 2 & 64 & NR & WT & + & + & - & - \\
\hline 3 & 16 & NR & WT & + & + & - & - \\
\hline 4 & $16 \dagger$ & NR & WT & + & + & - & - \\
\hline 5 & $>128$ & $B b s \mathrm{I}$ & $2143 \mathrm{G}$ & - & - & + & - \\
\hline 6 & 32 & $B b s \mathrm{I}$ & $2143 G$ & - & - & + & - \\
\hline 7 & 32 & $B b s \mathrm{I}$ & $2143 G$ & - & - & + & - \\
\hline 8 & $>128$ & $B b s \mathrm{I}$ & $2143 G$ & - & - & + & - \\
\hline 9 & 64 & $B b s \mathrm{I}$ & ND & - & - & + & - \\
\hline 10 & 64 & $B b s \mathrm{I}$ & ND & - & - & + & - \\
\hline 11 & $>128$ & $B b s \mathrm{I}$ & ND & - & - & + & - \\
\hline 12 & 32 & $B b s \mathrm{I}$ & ND & - & - & + & - \\
\hline 13 & $>128$ & $B b s \mathrm{I}+\mathrm{NR}$ & ND & - & + & + & - \\
\hline 14 & $>128$ & $B b s \mathrm{I}+\mathrm{NR}$ & ND & - & + & + & - \\
\hline 15 & 64 & $B b s \mathrm{I}+\mathrm{NR}$ & ND & + & - & + & - \\
\hline 16 & $>128$ & $B b s \mathrm{I}+\mathrm{NR}$ & $2143 \mathrm{G}$ & + & - & + & - \\
\hline 17 & $>128$ & BsaI & $2144 \mathrm{G}$ & - & - & - & + \\
\hline 18 & 16 & $B s a \mathrm{I}$ & $2144 \mathrm{G}$ & - & - & - & + \\
\hline 19 & 16 & BsaI & $2144 \mathrm{G}$ & - & - & - & + \\
\hline 20 & 8 & BsaI & $2144 \mathrm{G}$ & - & - & - & + \\
\hline 21 & 16 & $B s a \mathrm{I}$ & $2144 \mathrm{G}$ & - & - & - & + \\
\hline 22 & 16 & BsaI & ND & - & - & - & + \\
\hline 23 & 8 & BsaI & ND & - & - & - & + \\
\hline 24 & 32 & BsaI & ND & - & - & - & + \\
\hline 25 & 16 & BsaI & ND & - & - & - & + \\
\hline 26 & 16 & BsaI & ND & - & - & - & + \\
\hline 27 & 16 & BsaI & ND & - & - & - & + \\
\hline 28 & 4 & BsaI & ND & - & - & - & + \\
\hline 29 & 8 & BsaI & ND & - & - & - & + \\
\hline 30 & 4 & $B s a \mathrm{I}$ & ND & - & - & - & + \\
\hline 31 & $>128$ & BsaI & ND & - & - & - & + \\
\hline 32 & 64 & $B s a \mathrm{I}$ & $2144 \mathrm{G}$ & - & - & - & + \\
\hline 33 & $>128$ & BsaI + NR & ND & + & - & - & + \\
\hline 34 & 0.015 & NR & ND & + & - & - & - \\
\hline 35 & 0.015 & NR & WT & + & - & - & - \\
\hline 36 & 0.015 & NR & ND & + & - & - & - \\
\hline 37 & 0.007 & NR & WT & + & - & - & - \\
\hline 38 & 0.007 & NR & ND & + & - & - & - \\
\hline 39 & 0.007 & NR & ND & + & - & - & - \\
\hline 40 & 0.015 & NR & ND & + & - & - & - \\
\hline 41 & 0.007 & NR & ND & + & - & - & - \\
\hline 42 & 0.015 & NR & ND & + & - & - & - \\
\hline 43 & 0.06 & NR & ND & + & - & - & - \\
\hline 44 & 0.015 & NR & ND & + & - & - & - \\
\hline 45 & 0.06 & NR & ND & + & - & - & - \\
\hline 46 & 0.0035 & $\mathrm{NR}$ & WT & + & - & - & - \\
\hline 47 & 0.007 & NR & WT & + & - & - & - \\
\hline
\end{tabular}

MIC was determined by the agar dilution method. BsaI, PCR product restricted by $B s a \mathrm{I} ; B b s \mathrm{I}$, PCR product restricted by $B b s \mathrm{I}$. +, $\mathrm{OD}_{450}$ value higher than the cut off value;,$- \mathrm{OD}_{450}$ value lower than the cut off value.

${ }^{\star}$ Sequence analysis of the 23S rRNA gene revealed the genotype at residues 2143 and 2144 .

†MIC tested by Etest: $0.047 \mathrm{mg} / \mathrm{l}$ and presence of resistant mutants.

MIC, minimal inhibition concentration; PCR, polymerase chain reaction; RFLP, restriction fragment length polymorphism; WT, wild type; ND, not done; NR, not restricted. 
the confirmatory test; the results are reported in table 2. Both methods were applied to all strains. None of the 14 biopsy samples for which $H$ pylori culture was negative gave an $\mathrm{OD}_{450}$ higher than the cut off values with any of the probes (data not shown).

Of the 33 biopsy specimens from which resistant strains were isolated, 25 allowed the amplification of a $425 \mathrm{bp}$ fragment which hybridised with only one probe: one PCR product gave a positive signal with the probe detecting the A2143C mutation (biopsy sample 1), eight with the $2143 \mathrm{G}$ probe (samples $5-12$ ), and 16 with the $2144 \mathrm{G}$ probe (samples 17-32). The other eight samples yielded the amplification of a fragment hybridising with more than one probe: $2143 \mathrm{C}$ and wild type (samples 2, 3, and 4), 2143G and 2143C (samples 13 and 14), 2143G and wild type (samples 15 and 16), and 2144G and wild type (sample 33). The $\mathrm{OD}_{450}$ values were significantly higher than the cut off values needed to consider them as multiple genotypes. Fourteen samples from which clarithromycin susceptible strains were isolated gave a single positive signal with the probe detecting the wild type genotype (samples 34-47).

When the DEIA results were compared with those obtained by the reference methods, complete agreement between the different techniques was found. All the specimens classified as resistant on the basis of MIC gave a positive signal with a probe that detected a resistant genotype (specimens 1-33). Likewise, those containing susceptible strains were found to hybridise with the wild type probe (specimens 34-47). In one case (specimen 4), it should be noted that the MIC determined by Etest was $0.047 \mathrm{mg} / 1$ and some resistant mutants were observed. As expected, the MIC determined by the agar dilution method corresponded to a resistant strain.

Concerning the PCR/RFLP, the restriction by $B s a \mathrm{I}$ was always associated with hybridisation with the $2144 \mathrm{G}$ probe. A similar observation was made for the $2143 \mathrm{G}$ probe and the $B b s \mathrm{I}$ digestion. When two genotypes were present in the same biopsy specimen, the restriction profiles also correlated. The restriction enzyme assay was not able to discriminate between the A2143C genotype and the wild type genotype, as expected. Nevertheless, when mutant genotypes (A2143G and A2144G) were associated with either the A2143C or the wild type genotype, the restriction enzyme assay showed both genotypes (samples 13-16, and 33). Indeed, PCR/RFLP showed three bands of 331, 94, and $425 \mathrm{bp}$ corresponding to $B b s \mathrm{I}$ digestion products and an unrestricted fragment respectively (samples 13 to 16). For sample 33, three fragments were detected corresponding to $B s a \mathrm{I}$ restriction products (319 and $106 \mathrm{bp}$ ) and an undigested amplicon (425 $\mathrm{bp}$ ). In the case of specimens 34-47, the result of the DEIA correlated with that of the restriction enzyme assay; indeed, the PCR products were digested by neither $B b s \mathrm{I}$ nor $B s a \mathrm{I}$.

Sequence analysis of PCR products was performed in some samples of each mutant genotype (samples 1-8, 16-21, 32 35, 37, 46, and
47). In these cases, the sequence data confirmed the results provided by the other methods.

\section{Discussion}

Most of the guidelines published on the management of $H$ pylori infection such as the Maastricht Consensus Report ${ }^{1}$ and the Digestive Disease Health Initiation International Consensus Report ${ }^{2}$ do not recommend performing susceptibility testing before treating a patient for whom an $H$ pylori infection is diagnosed for the first time. This is largely because of the difficulty of performing susceptibility tests. Until now, culture was required and this technique is very demanding for the biologist; moreover, there is a long delay before the result is obtained. ${ }^{14}$ Although such standard susceptibility testing allows evaluation of all the antibiotics, in fact only susceptibility to macrolides, in particular clarithromycin, is really required because it is a major predictor of treatment failure. $^{3}$ Therefore the detection of clarithromycin resistant $H$ pylori strains will facilitate the choice of appropriate eradication treatments and the control of infection. Recently, in our laboratory, Pina et $a l^{10}$ developed a method which allows the detection of point mutations localised in the 23S rRNA gene (A2143C, A2143G, and A2144G) associated with clarithromycin resistance in $H$ pylori. This method based on hybridisation and immunoenzymic detection of the hybrids produced a result more quickly than the standard susceptibility tests, but primary culture was needed delaying the result by several days. In this study, we have shown that the technique can be applied directly to biopsy specimens, eliminating the need for $H$ pylori culture.

Although the cut off values have been previously published, ${ }^{10}$ it was necessary to determine them again for each probe because of the different nature of the samples. Indeed, the previous cut off values were lower than those determined in this study on biopsy specimens $(0.7,0.6,0.4$, and $0.5 v 0.9,0.96,0.6$, and 0.6 for wild type, $2143 \mathrm{C}, 2143 \mathrm{G}$, and $2144 \mathrm{G}$ probes respectively). However, the cut off value determined for the $2143 \mathrm{C}$ probe must be treated with caution because of the small number of samples tested. Testing of more specimens is necessary in order to include more with $H$ pylori strains harbouring the A2143C mutation, previously reported to be rare ${ }^{6}{ }^{15}$ and to validate the test further.

Of the 47 H pylori positive biopsy specimens, $39(83 \%)$ yielded a PCR product that hybridised with a single probe (specimens 1, 5-12, $17-32$, and 34-47 in table 2). This result shows that in these cases the mutation was homozygous. In the remaining cases, there may be two reasons for the multiple genotypes disclosed by DEIA. Firstly, the mutations may be heterozygous. Indeed, two copies of the $23 \mathrm{~S}$ rRNA gene exist in $H$ pylori chromosomal DNA, ${ }^{16}{ }^{17}$ and a mutation in one copy has been shown to be enough to confer clarithromycin resistance. ${ }^{8}$ Secondly, mixed infections with different $H$ pylori strains are known to occur in some people. ${ }^{18}$ Hence it is likely that some 
strains are clarithromycin resistant and others susceptible. In this study, we could not discriminate between these two situations. However, because of the apparent rarity of heterozygosity, ${ }^{48}$ the second hypothesis is most likely.

In the cases of multiple genotypes shown by DEIA, sequencing of the PCR products has not proved to be useful for elucidating the situation. Indeed, it is difficult to discriminate between a sequencing artefact and a genotype mixture, especially when different $H$ pylori strains are present in some biopsy specimens.

By applying the molecular method previously developed in our laboratory ${ }^{10}$ to detect clarithromycin resistance directly from biopsy specimens, we have confirmed that the point mutations A2143C, A2143G, and A2144G are associated with clarithromycin resistance in $H$ pylori, and that heterozygous mutations are sufficient to confer a high level of resistance. In our study, mutations at positions 2116 and 2142 recently described by Hultén et al ${ }^{\beta}$ were not found, nor any association between the level of resistance and the mutated genotype, in contrast with a recent report. ${ }^{19}$

Several characteristics of the PCR/DEIA method used in this study render it well adapted for routine work. Indeed, the use of a colorimetric hybridisation assay increases both the sensitivity of the PCR, as shown in other studies, ${ }^{12} 2021$ and the specificity because of the use of specific internal probes; furthermore, neither electrophoresis apparatus nor a UV light source is needed.

With regard to economical aspects, we do not have an exact value for the cost as it was an experimental study. However, given the expanding use of molecular tests in clinical bacteriological laboratories, it may be expected that the cost will be reasonable in the near future.

Finally, this procedure allows the detection of both the presence of $H$ pylori in gastric biopsy specimens and its resistance to macrolides, without the need for culture and within a day of endoscopy.

This study was supported by a grant from the Conseil Régional d'Aquitaine. $L \mathrm{M}$ was the recipient of a Junta Nacional de Investigaçâo Cientifica e Técnologico fellowship from Portugal.
1 European Helicobacter pylori Study Group. Current European concepts in the management of Helicobacter pylori pean concepts in the manag

2 The report of the Digestive Health Initiative International Update Conference on Helicobacter pylori. Gastroenterology 1997;113:S4-8.

3 Mégraud F. Resistance of Helicobacter pylori to antibiotics. Aliment Pharmacol Ther 1997;11:305-9.

4 Versalovic J, Shortridge D, Kibler K, et al. Mutations in $23 \mathrm{~S}$ rRNA are associated with clarithromycin resistance in Helicobacter pylori. Antimicrob Agents Chemother 1996;40: 477-80.

5 Debets-Ossenkopp YJ, Sparrius M, Kusters JG, et al. Mechnism of clarithromycin resistance in clinical isolates of Helicobacter pylori. FEMS Microbiol Lett 1996;142:37-42.

6 Occhialini A, Urdaci M, Doucet-Populaire F, et al. Macrolide resistance in Helicobacter pylori: rapid detection of point mutations and assays of macrolide binding to ribosomes. Antimicrob Agents Chemother 1997;41:2724-8.

7 Weisblum B. Erythromycin resistance by ribosome modification. Antimicrob Agents Chemother 1995;39:577-85.

8 Hultén K, Gibreel A, Sköld O, et al. Macrolide resistance in Helicobacter pylori: mechanism and stability in strains from clarithromycin-treated patients. Antimicrob Agents Chemother 1997;41:2550-3.

9 Arthur M, Molinas C, Mabilat C, et al. Detection of erythromycin resistance by the polymerase chain reaction using primers in conserved regions of erm rRNA methylase genes. Antimicrob Agents Chemother 1990;34:2024-6.

10 Pina M, Occhialini A, Monteiro L, et al. Detection of point mutations associated with resistance of Helicobacter pylori to clarithromycin by hybridization in liquid phase. $\mathcal{F}$ Clin Microbiol 1998;36:3285-90.

11 Sanger F, Nicklen S, Coulson AR. DNA sequencing with chain-terminating inhibitors. Proc Natl Acad Sci USA 1997;74:5463-7.

12 Monteiro L, Cabrita J, Mégraud F. Evaluation of performances of three DNA enzyme immunoassays for detection of Helicobacter pylori PCR products from biopsy specimens. Helicobacter pylori PCR products

13 Fauchère JL. Evaluation of the anti-H. pylori serum antibody response. In: Lee A, Mégraud F, eds. Helicobacter pylori: techniques for clinical diagnosis and basic research. London: Saunders, 1996;50-73.

14 Megraud F. A growing demand for Helicobacter pylori culture in the near future. Ital 7 Gastroenterol Hepatol 1997;29: $574-6$.

15 Stone GG, Shortridge D, Flamm RK, et al. Identification of 23S rRNA gene mutation in clarithromycin-resistant Helicobacter pylori. Helicobacter 1996;1:227-8.

16 Taylor DE, Ge Z, Purych D, et al. Cloning and sequence analysis of two copies of a $23 \mathrm{~S}$ rRNA gene from Helicobacter pylori and association of clarithromycin resistance with 23S rRNA mutations. Antimicrob Agents Chemother 1997;41:2621-8.

17 Tomb JF, White O, Kerlavage AR, et al. The complete genome sequence of the gastric pathogen Helicobacter pylori. Nature 1997;388:539-47.

18 Fantry GT, Zheng QX, Darwin PE, et al. Mixed infection with cag $A$-positive and cag $A$-negative strains of Helicobacter pylori. Helicobacter 1996;1:98-106.

19 Versalovic C, Osato MS, Spakovsky K, et al. Point mutations in the 23S rRNA gene of Helicobacter pylori associated with different levels of clarithromycin resistance. I Antimicrob Chemother 1997;40:283-6.

20 Zambardi G, Druetta A, Roure C, et al. Rapid diagnosis of Mycobacterium tuberculosis infections by ELISA-like detection of polymerase chain reaction products. Mol Cell Probes 1995;9:91-9.

21 Fujita SI, Lasker BA, Lott TJ, et al. Microtitration plate enzyme immunoassay to detect PCR-amplified DNA from Candida species in blood. F Clin Microbiol 1995;33:962-7. 